\title{
The Value of Apparent Diffusion Coefficient Measurement in Assessment and Follow Up of Multiple Sclerosis Patients
}

\author{
Samar Ramzy Ragheb*, Mena Elerian Youssef Ekladious
}

Department of Radiodiagnosis, Faculty of Medicine, Ain Shams University, Cairo, Egypt

*Corresponding author: Samar Ramzy Ragheb, Mobile: (+20)1003568254, E-mail: doc_mina2004@yahoo.com

\begin{abstract}
Background: It is widely accepted that multiple sclerosis effect on white matter extends beyond the demyelinating plaques and that the apparent diffusion coefficient (ADC) measurement can offer an insight on the effect of multiple sclerosis on normal appearing white matter.

Objective: The aim of the current work was to evaluate the role of ADC in assessment and follow up of multiple sclerosis patients.Patients and Methods: This retrospective study included a total of 20 healthy volunteers and 50 multiple sclerosis patients in different clinical subtypes, referred from Neurology Department and Outpatient Clinics, and conducted at Department of Radiodiagnosis, Ain Shams University Hospital. A follow up Magnetic resonance imaging (MRI) with ADC measurement was done every 6 months for 3 years.

Results: There was significant difference in the ADC of the normal appearing white matter of normal individuals and multiple sclerosis patients $(\mathrm{P}<0.0001)$. There was also significant difference in ADC measurement of plaques between relapsing remittent and chronic progressive cases $(\mathrm{P}<0.0001)$. The cutoff point in differentiating normal from multiple sclerosis patients was $0.8 \times 10-3 \mathrm{~mm} 2 / \mathrm{sec}$ with sensitivity $93.7 \%$, specifity $94.3 \%$ and accuracy $85 \%$. The cutoff point in differentiating relapsing remittent cases from progressive cases was $1.3 \times 10-3 \mathrm{~mm} 2 / \mathrm{sec}$ with sensitivity $89.3 \%$, specificity $85 \%$ and accuracy $80 \%$.

Conclusion: It could be concluded that routine measurement of the ADC value of the normal appearing white matter and multiple sclerosis plaques can help in assessment of the clinical subtype and shows high correlation with the degree of the disease progression.
\end{abstract}

Keywords: Diffusion, ADC, Multiple sclerosis.

\section{INTRODUCTION}

Multiple sclerosis (MS) is a chronic demyelinating disease that is characterized by white matter plaques that affects the brain and spinal cord ${ }^{(1-}$ 2). Axonal swelling, microglial activation and gliosis can also be signs of chronic injury of the normal appearing white matter ${ }^{(3)}$.

Although conventional MRI can show focal white matter demyelinating plaques yet due to its inability in detecting the damage of the normal appearing white matter, it shows week correlation with the clinical neurological deficit ${ }^{(4,5,6,7)}$.

There are several four clinical sub-types of multiple sclerosis the most common is relapsingRemitting (RRMS), (where the patients suffer from periods called relapses and exacerbations) ${ }^{(8)}$ and Secondary-Progressive (SPMS), in which symptoms worsen by time ${ }^{(9)}$.

Diffusion weighted MRI with ADC measurement plays an important role in assessment of early white matter changes. Water diffusion in white matter is highly directional because of the orientation of axons and the presence of myelin.

In cases of myelin damage and axon disruption the diffusivity along nerve fibers changes which is reflected on the ADC values ${ }^{(10,11,12)}$.
The aim of current study was to evaluate the role and value of ADC in assessment and follow up of multiple sclerosis patients.

\section{PATIENTS AND METHODS}

This retrospective study included a total of 20 healthy volunteers and 50 multiple sclerosis patients in different clinical subtypes, referred from Neurology Department and Outpatient Clinics, and conducted at Department of Radiodiagnosis, Ain Shams University Hospital. This study was conducted between February 2016 till January 2019.

\section{Ethical approval:}

This study was ethically approved by the Institutional review board and informed consents were taken from Ain Shams University and an informed written consent was taken from each participant in the study.

We excluded patients with MRI contraindication as those with cardiac pacemaker claustrophobia or cochlear implants.

\section{All patients were subjected to the following:}

This article is an open access article distributed under the terms and conditions of the Creative Commons Attribution (CC BY-SA) license (http://creativecommons.org/licenses/by/4.0/) 
1. Neurological examination: (Carried out by referring clinician)

\section{Imaging modalities:}

All the patients were examined with conventional and diffusion weighted MR imaging with comparison with the previous studies to evaluate the clinical course of the disease and to assess response to the medical treatment, using the same protocol of imaging.

Conventional Magnetic Resonance Imaging (cMRI): All patients underwent the MRI on 1.5 T Philips Achieva scanner MRI system using a 16-channel sensitivity-encoding (SENSE) head coil.

All cases were examined using the following protocol: - Axial T1-weighted images: $(\mathrm{TE}=15 \mathrm{~m} / \mathrm{sec} \mathrm{TR}=581$ $\mathrm{m} / \mathrm{sec})$.

- Axial fast spin-echo T2-weighted images: (TE=110 $\mathrm{m} / \mathrm{sec} \mathrm{TR}=4846 \mathrm{~m} / \mathrm{sec}$ ).

- Axial and sagittal Fluid-Attenuated InversionRecovery sequences (FLAIR): (TE=140 m/sec, $\mathrm{TR} / \mathrm{TI}=10000 / 2800 \mathrm{~m} / \mathrm{sec}$ ).

Diffusion weighted MR imaging: single shot spin echo EPI sequence (TR/TE/NEX: 3430/108 ms/I) with diffusion sensitivities of $b$ values $=0$ and $1000 \mathrm{~s} / \mathrm{mm}^{2}$.

Filed of vision $(\mathrm{FOV})=22 \mathrm{~cm}$ in axial images and $25 \mathrm{~cm}$ in sagittal images.

- Matrix (frequency x phase) 220x140

- Slice thickness $=5 \mathrm{~mm}$ with $1 \mathrm{~mm}$ interval. (In all sequences)

The ADC maps were calculated automatically by MRI software and included in the sequence. The interpretation of the images was done independently by two experienced radiologist with more than three years' experience in neuroimaging. Measurements of ADC were made in MS plaques and in the normal appearing white matter (in two different regions). The mean ADC values were calculated and expressed in $10^{-3} \mathrm{~mm}^{2} / \mathrm{sec}$. $\mathrm{ADC}$ values were measured in the normal white matter of 20 healthy control cases.

\section{Statistical analysis}

Recorded data were analyzed using the statistical package for social sciences, version 20.0 (SPSS Inc.,
Chicago, Illinois, USA). All quantitative data were expressed as mean (M) \pm Standard deviation (SD). Independent samples t-test was used to check differences in measurements. ROC curve was used to obtain the best cutoff point of ADC value. Parameters with $\mathrm{P}<0.05$ were considered significant and all tests were two tailed.

\section{RESULTS}

The study included 20 healthy volunteers $\{8$ males $(40 \%)$ and 12 females $(60 \%)\}$, their age ranged from 20 to 55 (mean age $=30 \mathrm{SD}=6$ ) and 50 multiple sclerosis patients in different clinical subtypes $\{22$ males $(44 \%)$ and 28 females $(56 \%)\}$, their age ranged from 20 to 57 (mean age $=33 \mathrm{SD}=5$ ). Patients were classified into two main groups healthy volunteers and multiple sclerosis patients with the patients further subdivided into two main subgroups (based on the duration of the disease and their clinical course), correlated with ADC value measurements; Group A: healthy volunteers, Group B multiple sclerosis patients who were sub classified into Group B1: chronic relapsing remittent cases (20 patients), Group B2: chronic progressive cases (30 patients) (Table 1).

Table (1): ADC values of the NWM in healthy volunteers versus NWM in MS patients.

\begin{tabular}{|c|c|c|c|}
\hline $\begin{array}{c}\text { Mean ADC } \\
\text { value } \\
(\mathrm{n}=70)\end{array}$ & $\begin{array}{c}\text { Healthy } \\
\text { volunteer } \\
(\mathrm{n}=20)\end{array}$ & $\begin{array}{c}\text { Multiple } \\
\text { sclerosis } \\
\text { patients } \\
(\mathrm{n}=50)\end{array}$ & \multirow{2}{*}{$\begin{array}{c}\mathbf{1}=\mathbf{6 . 3 2 8 7 7} \\
\mathbf{P}<\mathbf{0 . 0 0 0 0 1}\end{array}$} \\
\hline Min-Max & $0.5-0.9$ & $0.7-1.1$ & \\
\hline Mean \pm SD & $0.71 \pm 0.25$ & $0.88 \pm 0.45$ & \\
\hline
\end{tabular}

The mean ADC of the normal appearing white matter of the healthy volunteers (A) was $(0.71 \pm$ $\left.0.25 \times 10^{-3} \mathrm{~mm}^{2} / \mathrm{sec}\right)$. The mean ADC of the normal appearing white matter in multiple sclerosis patients was $\left(0.88 \pm 0.45 \times 10^{-3} \mathrm{~mm}^{2} / \mathrm{sec}\right)$.

The mean ADC value of the plaques at the relapsing remittent cases (B1) was (1.18 \pm $\left.0.23 \times 10^{-3} \mathrm{~mm}^{2} / \mathrm{sec}\right)$. The mean ADC value of the plaques at the chronic progressive cases (B2) was found to be elevated $\left(1.45 \pm 0.13 \times 10^{-3} \mathrm{~mm}^{2} / \mathrm{sec}\right)$ (Figure 1-4). 


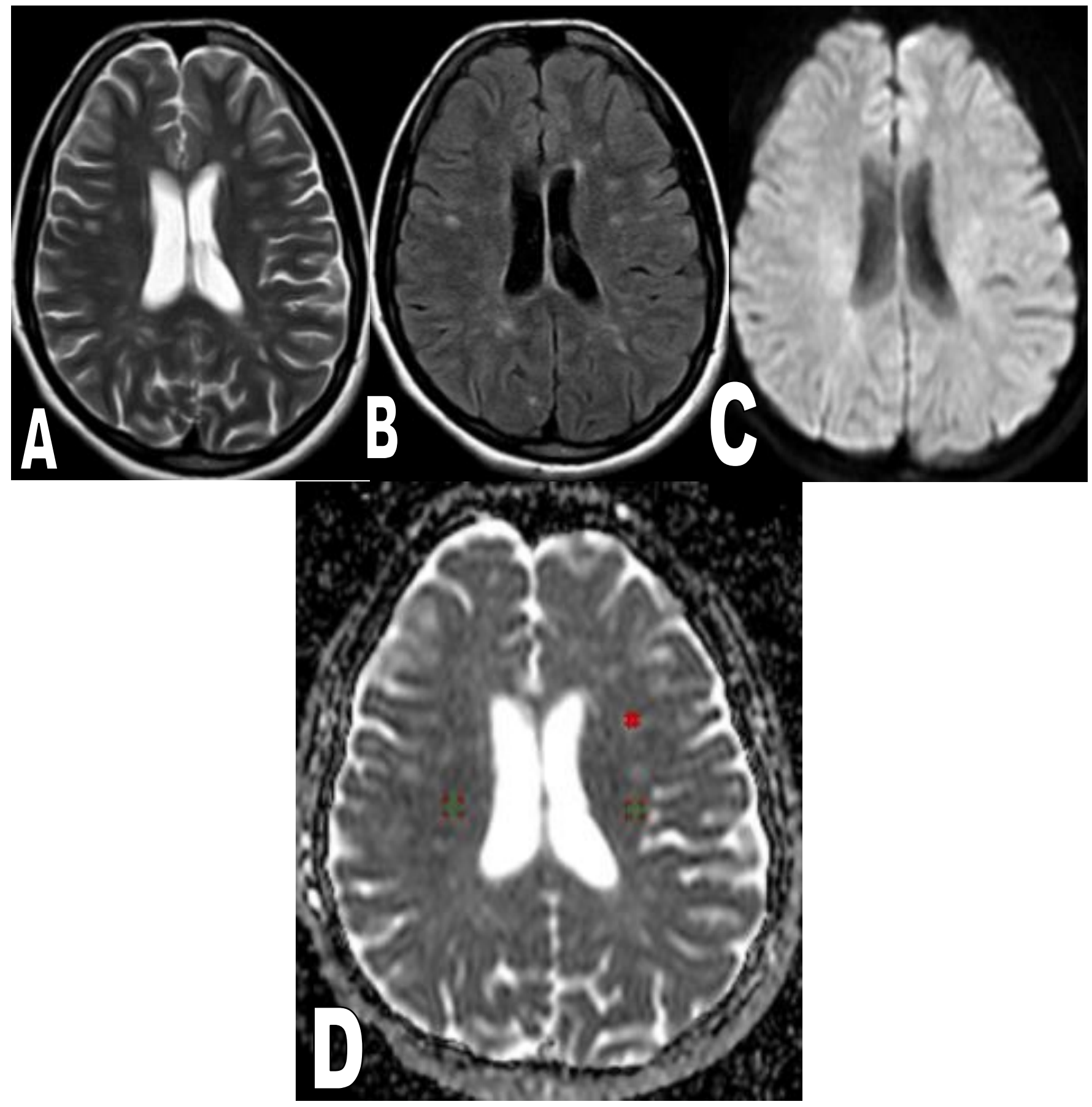

Fig. (1): 29 years old multiple sclerosis patient on relapsing remittent course, axial T2 and FLAIR WIs (A, B) shows multiple plaques with no diffusion restriction on DWI (C), Region of interest (ROI) are placed on normal appearing matter at the ADC map (D) with average ADC measurement: $0.9 \times 10^{-3} \mathrm{~mm}^{2} / \mathrm{sec}$, another ROI is placed on left periventricular plaque with average ADC measurement $1.2 \times 10^{-3} \mathrm{~mm}^{2} / \mathrm{sec}$. 


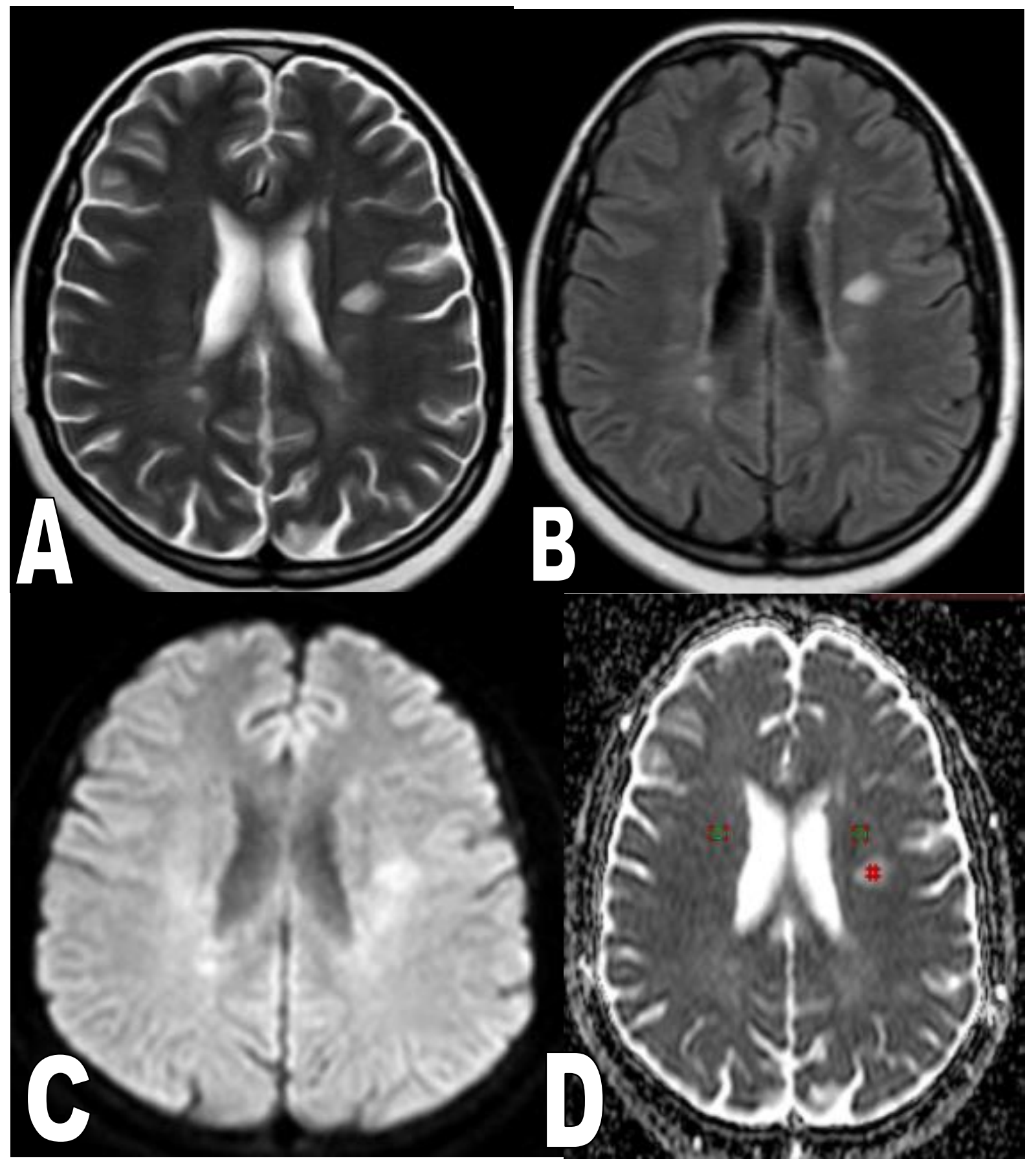

Fig. (2): 27 years old multiple sclerosis patient on relapsing remittent course, axial T2and FLAIR WIs (A, B) shows multiple plaques with no diffusion restriction on DWI (C), ROI are placed on normal appearing matter at the ADC map (D) with average ADC measurement: $0.95 \times 10^{-3} \mathrm{~mm}^{2} / \mathrm{sec}$, another ROI is placed on left periventricular plaque with average ADC measurement $1.3 \times 10^{-3} \mathrm{~mm}^{2} / \mathrm{sec}$. 


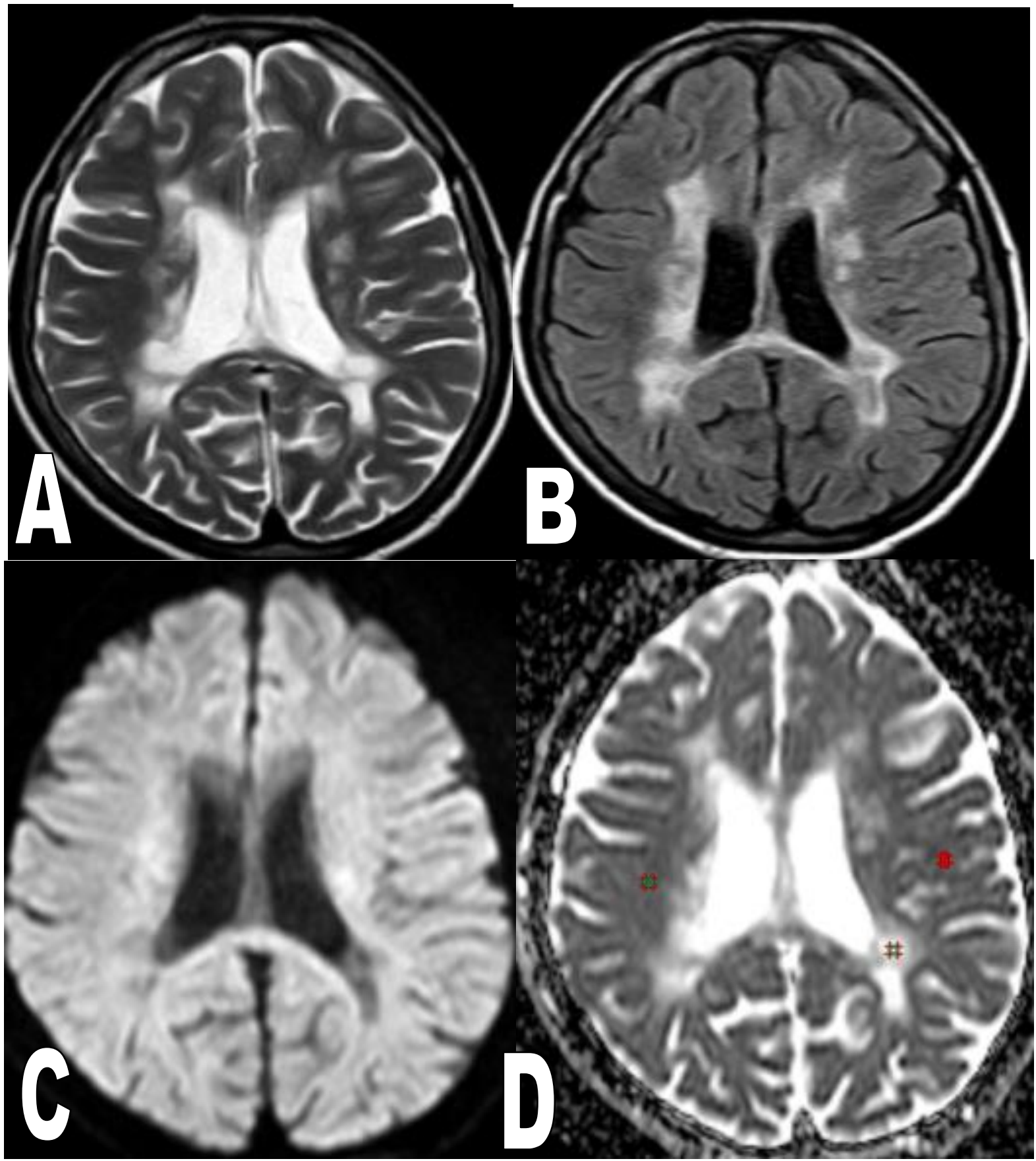

Fig. (3) 30 years old multiple sclerosis patient on progressive course, axial T2and FLAIR WIs (A,B) shows multiple plaques with no diffusion restriction on DWI (C), ROI are placed on normal appearing matter at the ADC map (D) with average ADC measurement: $1.1 \times 10^{-3} \mathrm{~mm}^{2} / \mathrm{sec}$, another ROI is placed on left periventricular plaque with average ADC measurement $1.5 \times 10^{-3} \mathrm{~mm}^{2} / \mathrm{sec}$. 


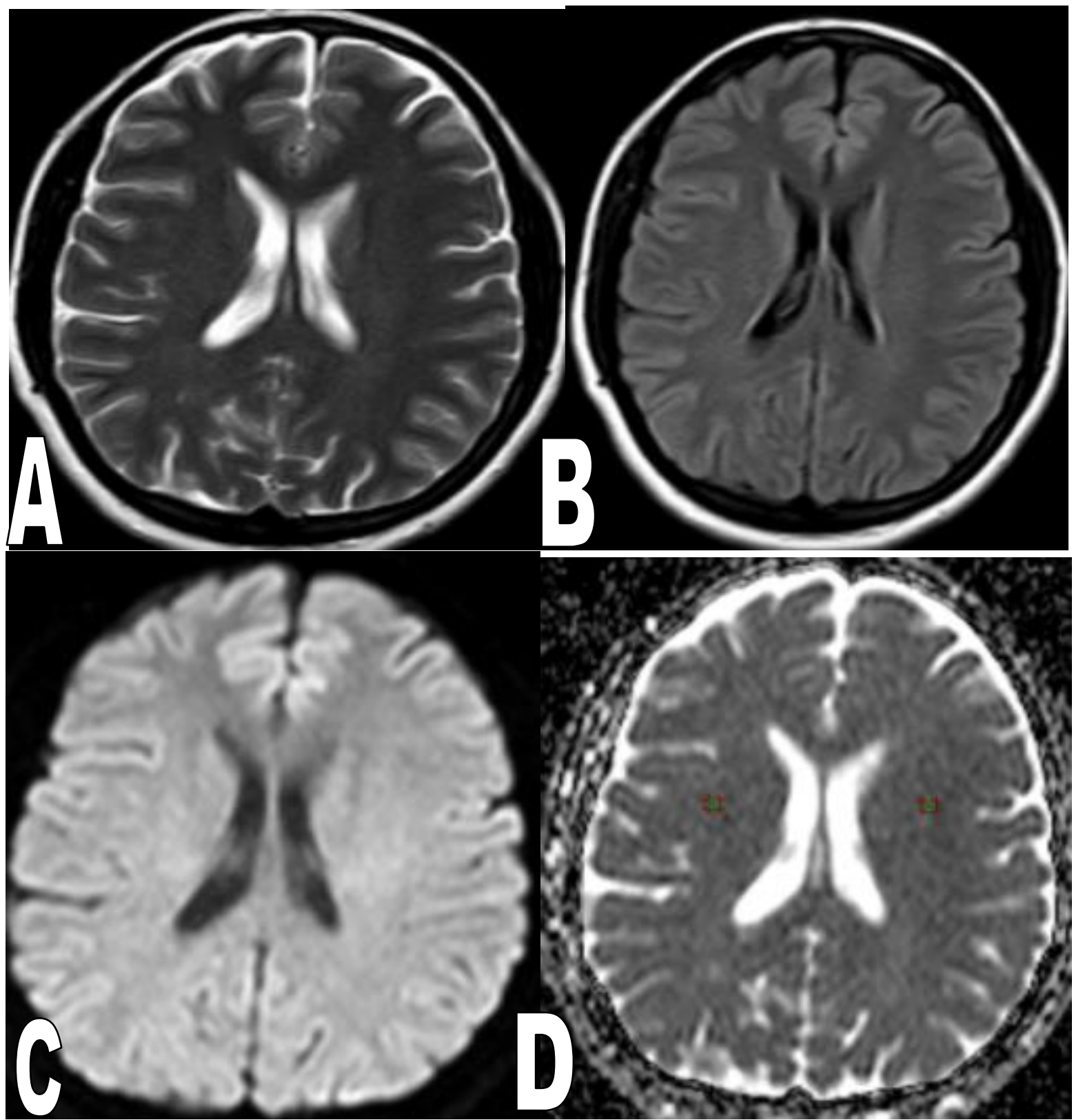

Fig. (4): 30 years healthy volunteer, axial T2, FLAIR WIs and DWI (A,B,C) shows normal appearance, ROI are placed on periventricular white matter at the ADC map(D) with average ADC measurement: $1.7 \times 10^{-3} \mathrm{~mm}^{2} / \mathrm{sec}$.

There was significant difference in the ADC of the normal appearing white matter of normal individuals and multiple sclerosis patients (Table 1). There was also significant difference in ADC measurement of plaques between relapsing remittent and chronic progressive cases (Table 2).

Table (2): ADC values of the developed plaques in different MS subtypes.

\begin{tabular}{|c|c|c|c|}
\hline $\begin{array}{c}\text { Mean ADC value } \\
\left(\mathrm{mm}^{2} / \mathrm{s}\right)(\mathrm{n}=50)\end{array}$ & $\begin{array}{c}\text { Relapsing remittent } \\
(\mathrm{n}=20)\end{array}$ & $\begin{array}{c}\text { Progressive } \\
(\mathrm{n}=30)\end{array}$ & \multirow{2}{*}{$\begin{array}{c}\mathbf{t}=\mathbf{7 . 2 9 1 0 9} \\
\mathbf{P}<\mathbf{0 . 0 0 0 0 1}\end{array}$} \\
\hline Min-Max & $1-1.3$ & $1.2-1.7$ & \\
\hline Mean \pm SD & $1.18 \pm 0.23$ & $1.45 \pm 0.13$ & \\
\hline
\end{tabular}

The cutoff point in differentiating normal healthy volunteers from multiple sclerosis patients was $0.8 \times 10^{-3} \mathrm{~mm}^{2} / \mathrm{sec}$ with sensitivity $93.7 \%$, specificity $94.3 \%$ and accuracy $85 \%$ (Table 3). 
https://ejhm.journals.ekb.eg/

Table (3): Mean ADC value and cutoff point in normal appearing white matter between healthy volunteers and multiple sclerosis patients.

\begin{tabular}{|c|c|c|c|c|c|}
\hline & AUC & Cutoff point & Sensitivity & Specificity & Accuracy \\
\hline $\begin{array}{c}\text { Mean ADC } \\
\text { value }\end{array}$ & 0.89 & 0.8 & $93.7 \%$ & $94.3 \%$ & $85 \%$ \\
\hline
\end{tabular}

The cutoff point in differentiating relapsing remittent cases from progressive cases was $1.3 \times 10^{-3} \mathrm{~mm}^{2} / \mathrm{sec}$ with sensitivity $89.3 \%$, specificity $85 \%$ and accuracy $80 \%$ (Table 4).

Table (4): Mean ADC value and cutoff point between relapsing remittent and progressive multiple sclerosis plaques.

\begin{tabular}{|c|c|c|c|c|c|}
\hline & AUC & Cutoff point & Sensitivity & Specificity & Accuracy \\
\hline $\begin{array}{c}\text { Mean } \\
\text { ADC value }\end{array}$ & 0.845 & 1.3 & $89.3 \%$ & $85 \%$ & $80 \%$ \\
\hline
\end{tabular}

\section{DISCUSSION}

Multiple sclerosis characterized by multiple attacks of activity followed by remission, thus the plaques after several such cyclic changes, show more demyelination with more destruction of both glial cells and axons, exerting expansion of the interstitial space and increased water content ${ }^{(\mathbf{1 3}, \mathbf{1 4})}$. Imaging plays a vital role in the diagnosis and follows up of multiple sclerosis patients yet neurological disability shows poor correlation with the findings in the conventional MRI as it cannot detect the underlying tissue damage ${ }^{(\mathbf{1 5})}$.

There is a persisting disagreement between clinical and magnetic resonance imaging results as the conventional MRI failed to formulate sufficient data regarding the disease extent and progression which eventually led to improper assessment of degree of disease process and had a negative effect in choosing the proper treatment regimen for the patients. In the recent years great effort has been dedicated to provide MR derived measurable values that are properly correlated with the histopathological changes. These include volumetry studies to measure the central nervous system atrophy, magnetization transfer imaging, proton MR spectroscopy and diffusion ${ }^{(16)}$.

The diffusion MR imaging is sensitive to detect increased water molecular motion that corresponds to these histologic changes ${ }^{(15)}$. Diffusion weighted (DW) images depends upon assessment of free molecular motion in vivo, with axon demyelination and damage this motion is disturbed and thus can be detected and measured by diffusion weighted images and ADC maps (16).

The current study showed that there was significant difference in the ADC of the normal appearing white matter of multiple sclerosis patients and healthy volunteers which highlights the tissue damage that was not detected by conventional MRI as axonal degradation and thus increase water diffusivity can be insufficient to produce signal changes at conventional
MRI yet it can be detected through the direct measurement of ADC value.

The current study also showed that there was a significant difference in the ADC measurement of plaques between the relapsing remittent and progressive clinical subtypes of multiple sclerosis as the ADC increases with the more extensive damage to the axon which occur at the patient with progressive course to the extent that it can reliably differentiate between the progressive and relapsing remittent clinical subtypes. Our study showed agreement with the study done by Tievsky et al. ${ }^{(17)}$ who found statistically significant difference between ADC values of normal appearing white matter in multiple sclerosis which was $0.79 \pm$ $0.11 \times 10^{-3} \mathrm{~mm}^{2} / \mathrm{sec}$ and the healthy volunteers which was $0.72 \pm 0.03 \times 10^{-3} \mathrm{~mm}^{2} / \mathrm{sec}$.

It also showed agreement with the study done by Christiansen $\boldsymbol{e t}$ al. ${ }^{(\mathbf{1 8})}$ as the mean ADC value was $0.77 \pm 0.08 \times 10^{-3} \mathrm{~mm}^{2} / \mathrm{sec}$ in the normal appearing white matter of multiple sclerosis patients while it was $0.71 \pm$ $0.1 \times 10^{-3} \mathrm{~mm}^{2} / \mathrm{sec}$ in the normal appearing white matter of the control cases.

Our results were in agreement with Castriota $\boldsymbol{e t}$ al. ${ }^{(19)}$ who found statistically significant differences in ADC values of the plaques between chronic relapsingremitting and chronic progressive MS groups ( $\mathrm{p}<$ 0.001 ), between normal white matter (NWM) of control group and normal white matter of the relapsingremitting and progressive MS groups ( $<<0.001)$. They reported that the mean value \pm SD of ADC was $(0.73 \pm$ $\left.0.02 \times 10^{-3} \mathrm{~mm}^{2} / \mathrm{s}\right)$ in NWM of healthy volunteers, $(0.95$ $\pm 0.08)$ in the plaques of relapsing-remitting MS, and $(1.435 \pm 0.129)$ in progressive MS.

Our results show disagreement with Roychowdhury et al. ${ }^{(20)}$ in the measurement of ADC values of the normal appearing white matter of MS cases as they reported no significant difference in the ADC values of NAWM as compared with that of NWM of a group of five healthy control cases $(p=0.18)$, this can 
be attributed to the relatively low sample of the healthy control groups.

The limitation of our study is that we did not perform high end specialized techniques as magnetization transfer and MR tractography in order to correlate the ADC values with them. Also we did not measure the ADC values of acute newly developed plaques as our study was concerned with the ADC measurement at chronic plaques in the relapsing remittent and progressive multiple sclerosis patients in order to better classify patient groups based on measurable data.

\section{CONCLUSION}

It could be concluded that routine measurement of the ADC value of the normal appearing white matter and multiple sclerosis plaques can help in assessment of the clinical subtype and shows high correlation with the degree of the disease progression.

\section{REFERENCES}

1. Kutzelnigg A, Lucchinetti C, Stadelmann $\mathrm{C}$ et al. (2005): Cortical demyelination and diffuse white matter injury in multiple sclerosis. Brain, 128(Pt 11):2705-12.

2. Frohman E, Racke M, Raine C (2006): Multiple sclerosis-the plaque and its pathogenesis. New England Journal of Medicine, 354 (9):942-55.

3. Ludwin $\mathbf{S}$ (2006): The pathogenesis of multiple sclerosis: relating human pathology to experimental studies. Journal of Neuropathology \& Experimental Neurology, 65 (4):305-18.

4. Khaleeli Z, Sastre-Garriga J, Ciccarelli O et al. (2007): Magnetisation transfer ratio in the normal appearing white matter predicts progression of disability over 1 year in early primary progressive multiple sclerosis. J Neurol Neurosurg Psychiatry, 78(10):1076-82.

5. Rovaris M, Bozzali M, Santuccio G et al. (2001): In vivo assessment of the brain and cervical cord pathology of patients with primary progressive multiple sclerosis. Brain, 124(Pt 12):2540-9.

6. McFarland H, Barkhof F, Antel J et al. (2002): The role of MRI as a surrogate outcome measure in multiple sclerosis. Multiple Sclerosis, 8(1):40-51.

7. Bar-Zohar D, Agosta F, Goldstaub D et al. (2008): Magnetic resonance imaging metrics and their correlation with clinical outcomes in multiple sclerosis: a review of the literature and future perspectives. Multiple Sclerosis, 14(6):719-27.
8. Hooper K (2011): Managing Progressive MS. National Multiple Sclerosis Society, (7): 173-7.

9. National Multiple Sclerosis Society (2011): NY multiple sclerosis: just facts. https://academic.oup.com/geronj/articleabstract/15/1/95/555389?redirectedFrom=fulltext

10. Nicola T, Silvia D, Tobias G et al. (2019): Evidence for Progressive Microstructural Damage in Early Multiple Sclerosis by Multi-Shell Diffusion Magnetic Resonance Imaging. Neuroscience, 403: 27-34.

11. Alexander A, Lee J, Lazar $M$ et al. (2007): Diffusion tensor imaging of the brain. Neurotherapeutics, 4: 316322.

12. Doughty C, Wang J, Feng W et al. (2016): FA Changes in Acute Stroke and Motor Outcome Predictions. Stroke, 47: 1520-1526.

13. Andrade R, Gasparetto E, Cruz L et al. (2007): Evaluation of white matter in patients with multiple sclerosis through diffusion tensor magnetic resonance imaging. Arquivos de Neuro-Psiquiatria, 65(3a): 561564.

14. Ragin A, Storey P, Cohen B et al. (2004): Whole brain diffusion tensor imaging in HIV-associated cognitive impairment. American Journal of Neuroradiology, 25(2): 195-200.

15. Andreadou E (2012): Neuroimaging in multiple sclerosis.

https://www.intechopen.com/books/neuroimagingclinical-applications/neuroimaging-in-multiple-sclerosis

16. Ramli N, Rahmat K, Azmi K et al. (2010): Past, present, and future of imaging in multiple sclerosis. J Clin Neurosci., 17:422-7.

17. Tievsky A, Ptak T, Farkas J (1999): Investigation of apparent diffusion coefficient and diffusion tensor anisotropy in acute and chronic multiple sclerosis lesions. AJNR Am J Neuroradiol., 20:1491-9.

18. Christiansen $P$, Gideon $P$, Thomsen $C$ et al. (1993): Increased water self-diffusion in chronic plaques and apparently normal white matter in patients with multiple sclerosis. Acta Neurol Scand., 87(3):195-9.

19. Castriota A, Tomaiuolo F, Sabatini U et al. (2000): Demyelinating plaques in relapsing-remitting and secondary-progressive multiple sclerosis: assessment with diffusion MR imaging. AJNR Am J Neuroradiol., 21(5):862-8.

20. Roychowdhury S, Maldjian J, Grossman R (2000): Multiple sclerosis comparison of trace apparent diffusion coefficients with MR enhancement pattern of the lesions. AJNR Am J Neuroradiol., 21:869-74. 